\title{
Comparison of Interferometry Techniques of Identification of Structures of Solid Media
}

\author{
By Carlos Orlando Jimenez Gonzalez \\ Juan Alejandro Vazquez Feijoo ${ }^{\dagger}$ \\ Jose Navarro Antonio ${ }^{*}$
}

This work presents a comparison between cross-correlation, crosscoherency and deconvolution in order to visualize their interrelations and possible performance under different circumstances of usage. Each interferometry method possesses its very own advantage. For example, although cross-correlation does not eliminate the source signature, it has the best performance for noisy signals. When arriving times are of primary importance, crosscoherence presents advantages as it is more precise though disregards the spectral amplitude.

\section{Introduction}

Seismic interferometry is a recent strategy used in several disciplines. The main purpose of seismic interferometry is to construct a Green's function between two locations from the records of earthquakes, microtremors and artificial sources. This powerful technique has been applied using three main algorithms: cross-correlation, deconvolution and cross-coherence; these methods have both advantages and disadvantages (Snieder et al., 2009). The seismic interferometry technique has been used successfully in previous works to extract the Green's function in layered medium using body waves from borehole array records. The use of record from borehole arrays in the algorithms of seismic interferometry implies 1D wave propagation.

Many problems in seismology can be explained by representing the Earth as a media defined by layers of certain thicknesses and mechanical properties (Haskell, 1953). Under certain considerations the seismic response of some alluvial valleys can be explained using a one-dimensional approach (Pujol, 2002), such the case of the valley of Mexico, where the geological configuration of subsoil favors the use of simple 1D propagation models (Reinoso, 1996). Although, one-dimensional geometry seems far from reality, such geometry is often seen in seismological observations by vertical arrays of seismographs in the shallow subsurface. One-dimensional geometry is enough

\footnotetext{
${ }^{*}$ PhD Student, CIIDIR, Oaxaca, National Polytechnic Institute, Mexico.

${ }^{\dagger}$ Senior Reader, CIIDIR, Oaxaca, National Polytechnic Institute, Mexico.

${ }^{*}$ Lecturer, CIIDIR, Oaxaca, National Polytechnic Institute, Mexico.
} 
if an appropriate combination of receivers and earthquakes are selected (Nakahara, 2006).

Here, the equations governing the displacements in a model of a layered medium are used to characterize the theoretical response obtained with the three algorithms of seismic interferometry, in the frequency and time domain. Additionally, the displacement equations are used in the generation of synthetic seismograms used to characterize the wavefield between two records. One advantage of a 1D model is that it allows us to visualize in simple way the relationships between these interferometry techniques and also allows us to analyze the accuracy and convenience of the usage of each technique under different conditions and for different objectives.

The first section analyzes the three techniques from a mathematic point of view, highlighting their direct physical dependency with cross-correlation. Second section makes use of the displacement equations for vertical propagation of plane $\mathrm{SH}$-waves in a single layered medium in order to apply the three interferometry techniques. The mathematical operations are developed in frequency domain and the interferometry responses are calculated using the surface displacement as the reference position. In order to obtain a better interpretation, the interferometry response is transformed to time domain using the Fourier transform. The equations of displacement in the surface and the bottom of the layer are used to construct the synthetic seismograms according with the convolutional model. The noise influence is evaluated when comparing the displacement field obtained for when uncorrelated random noise is added or not. In the particular case of cross-coherency the solution is obtained using a series expansion of the sign function of a cosine function, allowing the response as an infinite sum of Dirac delta impulses to be expressed.

\section{Mathematical Expressions for Interferometry Techniques}

In this section, a theoretical analysis and comparison between three used algorithms for retrieving the Green's function, is made. The simplest model of a layered medium used here consists of a horizontal single layer over a halfspace (Figure 1), the medium is both homogeneous and isotropic. Layers extend infinitely in the horizontal direction. In a lossless medium, properties of the layer and the half-space are determinate by the $\mathrm{SH}$ wave-velocity, $\beta_{i}$, and the mass density, $\rho_{i}$, where the subscript stands for the layer $(i=1)$ and the half-space ( $\mathrm{i}=2$ ). If the attenuation is considered, then the quality factor $Q_{i}$ is introduced. Displacements in the layer and the half-space are defined by $w_{1}(t)$ and $w_{2}(t)$. The free surface is in $y=0$ and the positive direction is downwards.

First of all, let us establish the algorithms used in seismic interferometry. The ground motion in a specific location can be described by a seismic source and the Green's function of the medium in such a location. The convolutional 
model establishes the dependency of a displacement at a point of the medium as function of the displacement of another point which is a real or virtual source. Then, if attenuation, transmission losses, noise and multiples are excluded, the seismic trace can be constructed by the 1D convolutional model $s * f$, where $*$ means convolution, $f$ is the primary reflectivity series and $s$ is the source wavelet.

In the following development, it is assumed that the earthquake source is far away from the record stations, therefore it is safe to assume that the incoming wavefield is a vertical plane wave (Figure 1a). Only one degree of freedom is assumed. For the model presented in Figure 1 the spectra of the wavefield recorded by the receiver on the surface at the location $\left(y=y_{0}=0\right)$ is given by,

$$
W_{0}(\omega)=S(\omega) G\left(y_{S}: y_{0}, \omega\right),(1)
$$

in this equation $S(\omega)$ is the called source signature, i.e., source spectrum, for the seismic source located at the point $y_{S}$ (Figure 1a) and may include effects such as scattering; the function $G\left(y_{S}: y_{0}, \omega\right)$ is the primary reflectivity from the source to the free surface.

The receiver in the location $y_{b}$ denotes a receiver located at any $y_{b}$ in the borehole. The recorded wavefield in this position is produced because of the source located in $y_{S}$. Therefore, this wavefield can be predicted by,

$$
W_{b}(\omega)=S(\omega) G\left(y_{S}: y_{b}, \omega\right),
$$

where $\omega$ is the angular frequency and $G\left(y_{S}: y_{b}, \omega\right)$ is the Green's function from the source position $y_{S}$ to $y_{b}$. As a reminder of this work, for simplicity, the argument is obviated, i.e. $G_{b}(\omega)=G\left(y_{S}: y_{b}, \omega\right)$ and $G_{0}=G\left(y_{S}: y_{0}, \omega\right)$, capital letters indicate the Fourier transform of the function.

Figure 1. Geometry of the Single Stratified Layer, Black Triangles Represent the Surface Receiver and the Borehole Receiver

a)
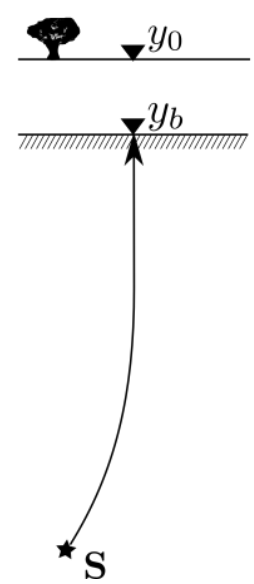

b)

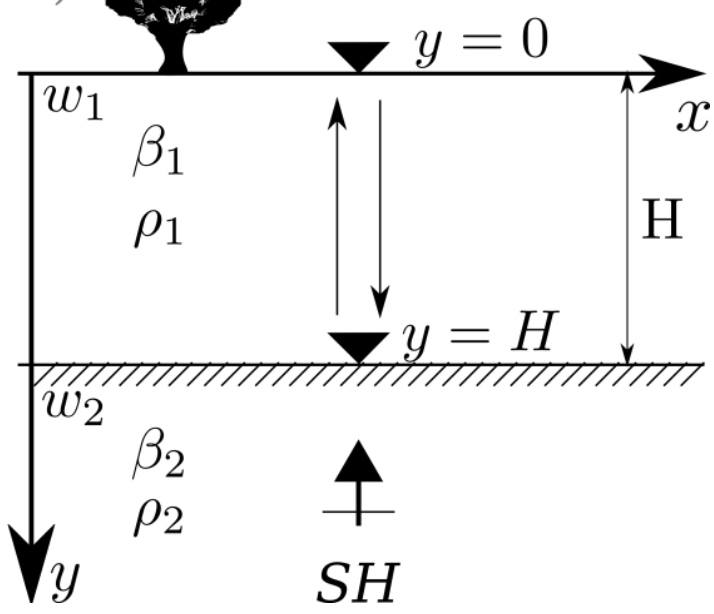




\section{Cross-Correlation}

The most simple seismic interferometry technique is cross-correlation (Wapenaar et al., 2010). A correlation in the frequency domain means a simple multiplication, then,

$$
C_{b, 0}=W_{b} W_{0}^{*}=|S|^{2} G_{b} G_{0}^{*},(3)
$$

the symbol $*$ denotes the complex conjugate. The cross-correlation between two receivers can also be expressed as a function of the Green's function between them,

$$
C_{b, 0}=|S|^{2} G_{b, 0},(4)
$$

where $G_{b, 0}$ is the plane wave Green's function between $y_{b}$ and $y_{0}$. Comparing equations (3) and (4) it is clear that $|S|^{2}$ can be considered the power spectra of a virtual source signature located at $y_{0}$. Allowing an abuse of nomenclature, for simplicity any real or virtual source is going to be denote by $S$.

The main limitation of convolution as can be seen from (4) is that does not allow the isolation of Green's function.

\section{Deconvolution}

A very simple way to extract from the convolution the Green's function is to suppose that both signals in equation (4) can be separated, then one may have

$$
G_{b, 0}=\frac{C_{b, 0}}{|S|^{2}},(5)
$$

equation (3) on equation (5) gives, $G_{b, 0}=W_{b} W_{0}^{*} /|S|^{2}$. Expanding the convolution operation in the numerator and by the algebraic manipulation one obtains,

$$
\frac{|S|^{2} G_{b, 0}}{|S|^{2}}=\frac{W_{b} W_{0}^{*}}{W_{0} W_{0}^{*}},(6)
$$

and the Green's function is finally isolated, equation (6) can be expressed as (Vasconcelos \& Snieder, 2008)

$$
D_{b, 0}=\frac{W_{b} W_{0}^{*}}{W_{0} W_{0}^{*}}=G_{b, 0},(7)
$$

Equation (7) provides a second technique for retrieving the Green's function, which is called deconvolution. This operation eliminates the power spectrum of the source (signature). This is an advantage in passive interferometry (using background noise or earthquake data) because the imprint waveform is different for each time data.

\section{Cross-Coherency}

On the other hand, some of practical applications are related to the Green's function angle phase, e.g. arriving times. Therefore, the amplitudes are 
neglected. Amplitudes from a signal close to the natural frequencies of the media are difficult to see, therefore this amplitudes may be meaningless. For this case, there is another way to deal with equation (3).

Let us divide equation (4) by the magnitudes of the signals in both points,

$$
\frac{|S|^{2} G_{b, 0}}{\left|W_{b}\right|\left|W_{0}\right|}=\frac{W_{b} W_{0}^{*}}{\left|W_{b}\right|\left|W_{0}\right|},
$$

developing the left hand side of the equation, one obtains

$$
\frac{W_{b} W_{0}^{*}}{\left|W_{b}\right|\left|W_{0}\right|}=\frac{|S|^{2} G_{b, 0}}{\sqrt{S G_{b, 0} S^{*} G_{b, 0}^{*} S S^{*}}} .
$$

the denominator can be reordered and then separated in two square roots, that can be expressed in terms of the magnitudes,

$$
\frac{|S|^{2} G_{b, 0}}{|S|^{2} \sqrt{G_{b, 0} G_{0}^{*}}}=\frac{G_{b, 0}}{\left|G_{b, 0}\right|}, \text { (10) }
$$

the signal obtained is the Green's function $G_{b, 0}$ between these points scaled to the unity, again the power spectrum of the source signature disappears. This algebraic treatment gives the equation for the third interferometry method called cross-coherency $\gamma_{i, j}$

$$
\gamma_{b, 0}=\frac{W_{b} W_{0}^{*}}{\left|W_{b}\right|\left|W_{0}\right|} .
$$

The use of the cross-coherency in seismic interferometry can be found, for example, in Prieto et al., (2009) and Nakata \& Snieder (2011) and demonstrates that cross-coherency which is proportional to the Green's function. Equation (10) indicates that cross-coherency extracts the spectrum of the normalized Green's function. If an actual linear relationship between both signals exists it results in a complex vector with constant unitary magnitude. Therefore cross-coherency only maintains the phase angle of the Green's function. Some authors consider equation (11) as a normalization of the cross spectrum referring it as complex coherency (Prieto et al., 2009).

According to definition of the $\operatorname{sgn}(x)=x /|x|$ the cross-coherency can also be obtained by

$$
\gamma_{b, 0}=\operatorname{sgn}\left(D_{b, 0}\right)
$$

\section{Interferometry Response of a Single Stratified Layer Model}

Here, a model of a single layered medium is used to characterize the response in the time domain of the traces obtained by the interferometry techniques. A model of this kind has been used to model some basic behavior in some structures (Somerville et al., 1991) and in some structures like buildings (Nakata \& Snieder, 2013). Considering that $W_{1}(0, \omega)=W_{0}$ and 
$W_{1}(H, \omega)=W_{H}$ are the displacement spectra in the free surface $y=0$, and in the top of the half-space $y=H$, respectively (see Figure $1 \mathrm{~b}$ ). $H$ is the thickness of the single layer, $W_{1}(y, \omega)=W_{y}$ is the displacement spectrum at any point inside the layer $(0 \leq y \leq H)$. In addition, the model has a linear response and no losses are considered. The mechanical properties of the layer and the half space are $\beta_{1}, \rho_{1}$ and $\beta_{2}, \rho_{2}$, respectively. The subscripts 1 and 2 stand for the layer and the half space. Perfect welded is the boundary condition between the soft layer and the half space (continuity of displacements and tractions), and at $y=0$ the free-stress condition. The incident wave in the lower medium is a plane SH-wave that propagates along the y-axis of the type $e^{i \omega\left(t+\frac{y}{\beta_{1}}\right)}$.

The geometry considered here is a special case of the proof by Wapenaar et al., (2004) for application on seismic interferometry. For convenience, the displacement in the surface is considered the source signature and it is governed by,

$$
W_{0}=S=\frac{2 A_{0} e^{-i k_{2} H}}{\cos \left(\frac{H \omega}{\beta_{1}}\right)+i \eta \sin \left(\frac{H \omega}{\beta_{1}}\right)}
$$

where $A_{0}$ is the incident amplitude, commonly considered like $A_{0}=1$, $i=\sqrt{-1}, \eta=\beta_{1} \rho_{1} / \beta_{2} \eta_{2}$ is the impedance contrast between the layer and the half-space.

The of power spectrum of $S$ is

$$
|S|^{2}=\frac{4 A_{0}^{2}}{\cos ^{2}\left(\frac{H \omega}{\beta_{1}}\right)+\eta^{2} \sin ^{2}\left(\frac{H \omega}{\beta_{1}}\right)},
$$

that is equal to the square of the amplification factor in the free-surface (for a single strata layer of thickness $H$ ). The spectral displacement in $y=H$ is,

$$
W_{H}=S \cos \left(\frac{H \omega}{\beta_{1}}\right)
$$

By the use of equation (3) the cross-correlation is,

$$
C_{H, 0}=\frac{|S|^{2}}{2}\left(e^{\frac{i \omega H}{\beta_{1}}}+e^{-\frac{i \omega H}{\beta_{1}}}\right) .
$$

The deconvolution (equation (7)) for displacement at any position $y \leq H$ is,

$$
D_{y, 0}=\frac{1}{2}\left(e^{\frac{i \omega y}{\beta_{1}}}+e^{-\frac{i \omega y}{\beta_{1}}}\right),
$$

in particular when $y=H$ is,

$$
D_{H, 0}=\frac{W_{H} W_{0}}{W_{0} W_{0}}=\frac{1}{2} e^{\frac{i \omega H}{\beta_{1}}}+\frac{1}{2} e^{-\frac{i \omega H}{\beta_{1}}}
$$


In all of these equations attenuation and dispersion are discharged. The two waves produced are symmetrical and the energy is equally distributed. Similar results are obtained elsewhere, e. g. Trampert et al. (1993) and Snieder \& Şafak (2006) in buildings.

Applying the inverse Fourier on equation (18) the impulse response at $y=H$ is obtained (when the unitary impulse is applied at $y=0$ ),

$$
D_{H, 0}(t)=\frac{1}{2}\left[\delta\left(t-\frac{H}{\beta_{1}}\right)+\delta\left(t+\frac{H}{\beta_{1}}\right)\right]
$$

The impulse response is composed by an upgoing wave traveling from the bottom receiver to the surface receiver collapsing in $t=0$ and an down-going wave traveling from the surface to the half-space (Snieder \& Şafak 2006),

Transforming equation (16) into the time domain one obtains,

$$
\int_{-\infty}^{\infty} w(0, \tau) w(H, t+\tau) d \tau=\frac{S(t)}{2} *\left(\delta\left(t-\frac{H}{\beta_{1}}\right)+\delta\left(t+\frac{H}{\beta_{1}}\right)\right)
$$

where $S(t)$ is the autocorrelation of the source signal $s(t)$, i.e., $S(t)=s(t) * s(t)$. For any position $y \leq H$ one has

$$
\int_{-\infty}^{\infty} w(0, \tau) w(H, t+\tau) d \tau=\frac{S(t)}{2} *\left(\delta\left(t-\frac{y}{\beta_{1}}\right)+\delta\left(t+\frac{y}{\beta_{1}}\right)\right)
$$

The two terms in equation (18) can be interpreted as the upward and downward wave travelling between the two receivers. The term $|S|^{2}$ in equation (14) is equivalent to the square of the well-known amplification factor of a single layered medium, consequently, the maximum amplification factor is equal to $4 / \eta^{2}$.

The value of $|S|^{2}$ introduces resonant frequencies known as scattered waves. This happens when $\omega H / \beta_{1}=\pi(2 n-1) / 2$; where $n \in \mathbf{N}$. This fact implies that the correlation exhibits these additional reflexions not observed in deconvolution. .

The modulus of the transfer function of a single layered medium is expressed by,

$$
\left|\frac{W_{0}}{W_{H}}\right|=\mid \cos \left(\frac{H \omega}{\beta_{1}}\right)^{-1} .
$$

On the other hand, the cross-coherency (equation (11)) yields,

$$
\gamma_{H, 0}(\omega)=\frac{1}{2}\left(e^{\frac{i \omega H}{\beta_{1}}}+e^{-\frac{i \omega H}{\beta_{1}}}\right) \cos \left(\frac{\omega H}{\beta_{1}}\right)^{-1}
$$

Equation (23) is interpreted as the Green's function over its module. In fact, this equation is a sign function of the deconvolution (equations (7) and (18)) and provides a function that resembles a square wave. This function can be expressed in an expanded form as follows, 


$$
\gamma_{H, 0}(\omega)=\operatorname{sgn}\left(\cos \left(H k_{1}\right)\right)=\frac{4}{\pi} \sum_{n=1}^{\infty} \frac{(-1)^{n-1}}{2 n-1} \cos \left(\frac{(2 n-1) \omega H}{\beta_{1}}\right),
$$

applying the inverse Fourier Transform on the expression (24) results in,

$$
\gamma_{H, 0}(t)=\frac{2}{\pi} \sum_{n=1}^{\infty} \frac{(-1)^{n-1}}{2 n-1}\left[\delta\left(t+\frac{(2 n-1) H}{\beta_{1}}\right)+\delta\left(t-\frac{(2 n-1) H}{\beta_{1}}\right)\right],
$$

where $n$ is the number of times that the energy is reflected.

The result obtained by cross-coherency gives the same phases in the two first spikes than those obtained by cross-correlation or deconvolution. Additional spikes in equation (26) portray the phases of the multiples produced by the standing wave in the soft layer. However, it must be observed that even if the displacements are described at any other point, i.e., $y \neq H$, multiples still appear; this is an important unphysical effect. Cross-coherence produces multiples at any point where the data is taken. Then, these additional impulses are attributed to an unphysical boundary created by the cross-coherency operation.

Equations (16), (17) and (23) demonstrate the relationship between the three interferometry techniques for retrieving the impulse response from a single stratified layer. From the analysis of the mentioned expressions the following asseverations are proved: 1) cross-correlation gives the Green's function convolved with the source signature. 2) Deconvolution extracts the Green's function as it takes away the source signature. 3) Cross-coherency is a normalized version of deconvolution. As cross-coherency is a normalized process, the useful data comes mainly from the phase angle. For many purposes the magnitude is meaningless or difficult to obtain with practical accuracy, so cross-coherency may provide a good alternative. Nevertheless, cross-coherency presents other problems; it produces harmonics as a reflection on a virtual frontier at any point the data is taken hindering the interpretation.

Another big issue with the interferometry techniques is the influence that the noise has on them. In order to illustrate the noise effect on the techniques of seismic interferometry in the case analyzed above, it is presented as a numerical example. For this purpose, equations (13) and (15) are used to generate synthetics seismograms in two positions as is illustrated in Figure 1b. Synthetic seismograms are made using the convolutional model for the cases: of noise-free and adding uncorrelated noise. Equations (13) and (15) are convolved with a Ricker wavelet to obtain the displacement spectra and transformed to the time domain by the inverse fast Fourier transform (IFFT). In the simulation a Ricker wavelet of $5 \mathrm{~Hz}$ is used, the layer's mechanical properties are $\beta_{1}=0.7 \mathrm{~km} / \mathrm{s} \rho_{1}=0.7$, when attenuation is considered, and in the half-space $\beta_{2}=1.2 \mathrm{~km} / \mathrm{s}, \rho_{2}=1.2$ and $Q_{2}=500$.

On the left side of Figure 2 the traces without attenuation are shown, the acausal (up going waves) and causal pulses (down going waves) are symmetric; on the right side of Figure 2 the results are plotted including non-correlated random noise in the convolutional model and attenuation is induced by the wavenumber in the displacements, is evident that the symmetry of amplitudes 
is lost. Symmetry is recover using the real part of the interferometry traces, where effects like attenuation is discarding and helps to identify the arrival times. In the presence of uncorrelated noise the cleanest waveforms are obtained with cross-correlation. The multiples obtained in the crosscorrelograms have the same phases that the cross-coherency in the bottom of the layer. It's evident that the usefulness of cross-correlation is related to the fact of obtaining a less distorted signal.

Figure 2. Interferometry Traces from Synthetic Seismograms. Deconvolution, Cross-Correlation and Cross-Coherency of the Displacement in the Bottom of the Soft Layer as a Function of the Displacement Recorded in the Free Surface. On the Left without Attenuation, on the Upper Right Side with Attenuation and Uncorrelated Additive Noise. In the Bottom Right Side with Additive Noise but is only Transform the Real Part of the Spectrum
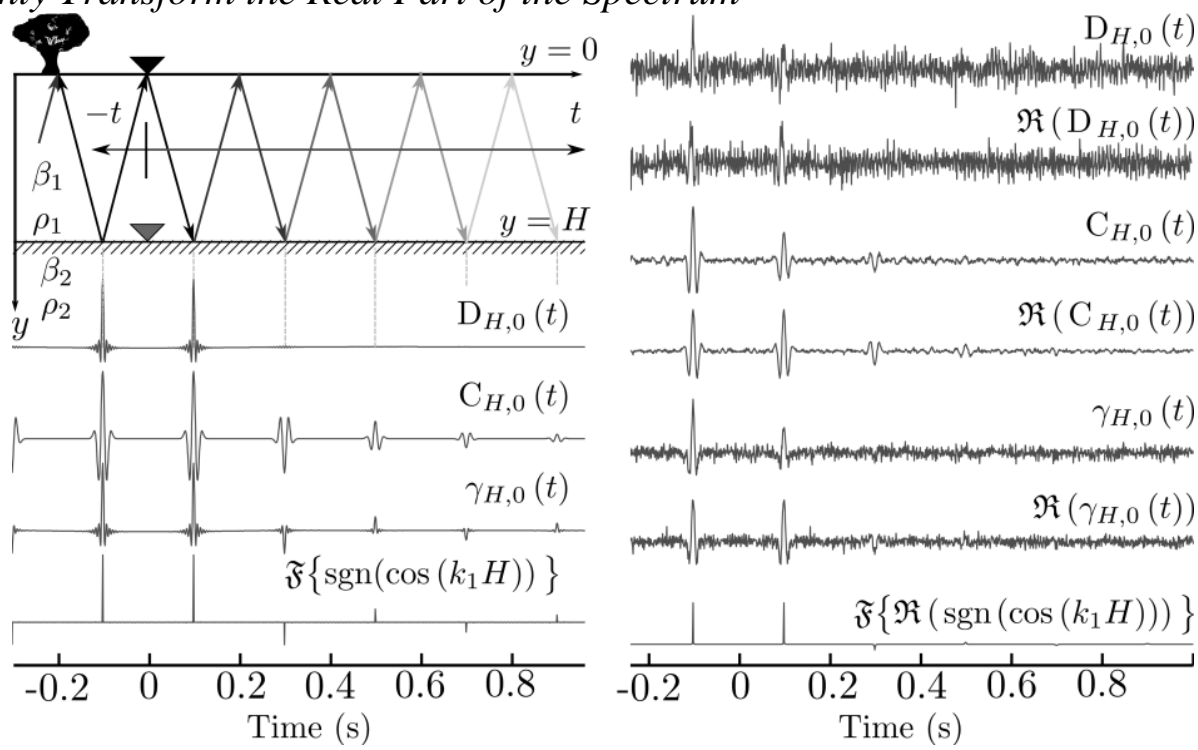

\section{Conclusions}

This work provides a point of comparison of the good and bad characteristics of each interferometry technique according to the usage of the information. This is a good means to evaluate the best interferometry technique for the specific purpose. Cross-correlation is the best for noisy signals; however the source signature is still immersed into the data. Deconvolution isolates the Green's function but has problems dealing with uncorrelated noise in signals. When arriving times are of primary importance, then crosscoherence is a good alternative, as only the phase is accurately obtained however this technique is not good for noisy signals and produces spurious components on the site data. 


\section{References}

Haskell, N. A., 1953. The dispersion of surface waves on multilayered media. Bulletin of the Seismological Society of America, 43(1), pp.17-34.

Nakahara, H., 2006. Theoretical background of retrieving Green's function by crosscorrelation: one-dimensional case. Geophysical Journal International, 165(3), pp.719-728.

Nakata, N. \& Snieder, R., 2013. Monitoring a Building Using Deconvolution Interferometry. II: Ambient-Vibration Analysis. Bulletin of the Seismological Society of America, 104(1), pp.204-213.

Nakata, N. \& Snieder, R., 2011. Near-surface weakening in Japan after the 2011 Tohoku-Oki earthquake. Geophysical Research Letters, 38(17), p.L17302.

Prieto, G. A., Lawrence, J.F. \& Beroza, G.C., 2009. Anelastic Earth structure from the coherency of the ambient seismic field. Journal of Geophysical Research, 114(B7), p.B07303.

Pujol, J., 2002. Unexpected Values of Qs in the Unconsolidated Sediments of the Mississippi Embayment. Bulletin of the Seismological Society of America, 92(3), pp.1117-1128.

Reinoso, E., 1996. Two and three dimensional scattering from topographical structures and alluvial valleys and comparisons with accelerometric data: the case of Mexico city. In Eleventh World Conference on Earthquake Engineering. Elsevier Science Ltd.

Snieder, R. et al., 2009. A Comparison of Strategies for Seismic Interferometry. Surveys in Geophysics, 30(4-5), pp.503-523.

Snieder, R. \& Şafak, E., 2006. Extracting the Building Response Using Seismic Interferometry: Theory and Application to the Millikan Library in Pasadena, California. Bulletin of the Seismological Society of America, 96(2), pp.586-598.

Somerville, P.G. et al., 1991. The influence of site conditions on the spatial incoherence of ground motions. Structural Safety, 10(1-3), pp.1-13.

Trampert, J., Cara, M. \& Frogneux, M., 1993. SH Propagator Matrix and Qs Estimates From Borehole- and Surface-Recorded Earthquake Data. Geophysical Journal International, 112(2), pp.290-299.

Vasconcelos, I. \& Snieder, R., 2008. Interferometry by deconvolution: Part 1 Theory for acoustic waves and numerical examples. GEOPHYSICS, 73(3), pp.S115-S128.

Wapenaar, K. et al., 2010. Tutorial on seismic interferometry: Part 2 - Underlying theory and new advances. GEOPHYSICS, 75(5), pp.75A211-75A227.

Wapenaar, K., Thorbecke, J. \& Draganov, D., 2004. Relations between reflection and transmission responses of three-dimensional inhomogeneous media. Geophysical Journal International, 156(2), pp.179-194. 